\title{
Das Bildungssystem. Entwicklungen und Herausforderungen
}

\author{
Jacqueline Maria Radtke
}

Eine fortschrittliche Bildungs- und Sozialpolitik hat in den vergangenen Jahren zu positiven Entwicklungen des brasilianischen Bildungswesens geführt. Der Bevölkerungsanteil mit Zugang zur Schulbildung hat deutlich zugenommen, immer mehr Sekundarschulabsolventen streben einen Hochschulabschluss an. Trotzdem sieht sich die brasilianische Bildungspolitik nach wie vor mit großen Herausforderungen konfrontiert. Der folgende Beitrag stellt den Aufbau des Bildungssystems vor, analysiert die Entwicklungen seit den 1990er Jahren und skizziert aktuelle Probleme und Herausforderungen.

\section{Der Aufbau des Bildungssystems}

Laut Art. 6 der brasilianischen Verfassung ist Bildung ein soziales Grundrecht. Art. 227 bezeichnet Erziehung und Bildung als gemeinsame Aufgaben von Familie, Gesellschaft und Staat. Die gesetzlichen Grundlagen des gegenwärtigen Bildungssystems sind im Bundesbildungsgesetz von 1996 (Lei de Diretrizes e Bases da Educaşão - LDB) festgelegt. Das Bildungssystem zeichnet sich durch eine dezentrale Verteilung der Verwaltungs- und Verantwortungsbereiche aus. Auf der Ebene der Zentralregierung erarbeitet das Bildungsministerium (Ministério da Educação - MEC) alle zehn Jahre einen Nationalen Bildungsplan (Plano Nacional de Educação - PNE), in dem die Bildungsrichtlinien für die kommende Dekade festgelegt werden. Die Kontrolle und Evaluierung erfolgt zusammen mit dem Nationalen Bildungsrat (Conselho Nacional de Educação - CNE) (BMBF 2012). Die daran anschließende Ausgestaltung der Bundesbildungspolitik und die Sicherstellung eines konkreten Bildungsangebots in Form von Bildungseinrichtungen und Lehrplänen ist Aufgabe der bundesstaatlichen Bildungsministerien (Secretarias de Educação). Den Gemeinden und Städten kommt hierbei die Aufgabe der Sicherstellung eines Teils des Betreuungsund Bildungsangebots im vorschulischen Bereich und im Grundschulbe- 
reich zu (Perez/Fraga de Melo/Fichtner 2010: 624). Laut Bildungsgesetz ist es vorgesehen, dass die Zentralregierung mindestens $18 \%$ und die Bundesstaaten, Bundesdistrikte und Gemeinden mindestens $25 \%$ ihrer Steuereinnahmen "in die Versorgung und Entwicklung des öffentlichen Bildungssystems investieren" (Fritsche 2003: 53).

Neben dem öffentlichen Bildungssystem besteht in Brasilien - wie in den meisten lateinamerikanischen Ländern - ein durch privat finanzierte Einrichtungen zur Verfügung gestelltes Bildungsangebot. Die Gründung und Unterhaltung privater Schulen ist seit 1988 erlaubt. Private Bildungseinrichtungen unterstehen jedoch der staatlichen Kontrolle. Sie müssen nachweisen, dass sie den "allgemeinen Normen der Erziehung" (Richter 2013: 182) entsprechen. Der private Bildungssektor setzt sich sowohl aus profitorientierten als auch aus gemeinnützigen Organisationen zusammen, wobei insbesondere im Bereich der höheren Bildung Kirchen als Träger gemeinnütziger privater Bildungseinrichtungen fungieren (Müller 2009: 12).

Die schulische Grundbildung (Educação Básica) setzt sich aus der Vorschulbildung (Educação Infanti), der Fundamentalbildung (Ensino Fundamental) und der mittleren Bildung (Ensino Médio) zusammen. Im Rahmen der Vorschulbildung können Kinder bis zum dritten Lebensjahr in Kindergärten betreut werden und vom vierten bis zum fünften Lebensjahr eine Vorschule besuchen. Dieses erste Bildungsangebot "soll der psychologischen, intellektuellen und sozialen Entwicklung dienen" (Fritsche 2003: 47, nach Art. 29, LDB). Die obligatorische neunjährige Fundamentalbildung dauert vom sechsten bis zum vierzehnten Lebensjahr. Sie unterteilt sich in den fünf Jahre andauernden Grundschulunterricht und die vierjährige untere Sekundarstufe. Neben der Vermittlung der Fähigkeiten des Lesens, Schreibens und Rechnens soll die Fundamentalbildung auch dazu dienen, die Schüler zu mündigen Bürgern zu erziehen. Hierfür werden Kenntnisse in den Bereichen Politik und Gesellschaft, Kunst und Technologie vermittelt. Die Fundamentalstufe legt die Grundlagen für einen weiteren Bildungsweg. Nach weiteren drei Schuljahren kann ein Abschluss der mittleren Bildungsstufe erreicht werden. Ziel der mittleren Bildung ist es, die in der Fundamentalstufe erworbenen Kenntnisse zu festigen und zu vertiefen. Die Schüler sollen lernen, mit neuen Situationen umzugehen, um auf eine bevorstehende berufliche Tätigkeit vorbereitet zu sein. Darüber hinaus sollen sie ihre Rechte und Pflichten als Bürger kennenlernen und sich in Kritikfähigkeit üben. Außerdem soll die Fähigkeit des Verbindens 
Das Bildungssystem. Entwicklungen und Herausforderungen

\section{Übersicht 1: Das brasilianische Bildungssystem}

\begin{tabular}{|c|c|c|c|}
\hline Alter & \multicolumn{3}{|l|}{ Bildungsstufe } \\
\hline $0-5$ & \multirow[b]{3}{*}{$\begin{array}{l}\text { Grundbildung } \\
\text { (Educaçáo Básica) }\end{array}$} & \multicolumn{2}{|c|}{$\begin{array}{l}\text { Vorschulbildung (Educaçáo Infantil) } \\
\text { Betreuung in Kindergärten bis zum 3. Lebensjahr } \\
\text { Besuch von Vorschulen vom 4. bis 5. Lebensjahr } \\
\text { Zuständigkeit: Gemeinden }\end{array}$} \\
\hline $6-14$ & & \multicolumn{2}{|c|}{$\begin{array}{l}\text { Fundamentalbildung (Ensino Fundamental) } \\
\text { 5-jährige Grundschulbildung (Klasse 1-5) } \\
\text { 4-jährige untere Sekundarschulbildung (Klasse 6-9) } \\
\text { Zuständigkeit: Gemeinden und Bundesstaaten }\end{array}$} \\
\hline $15-17$ & & $\begin{array}{l}\text { Mittlere Bildung } \\
\text { (Ensino Médio) } \\
\text { 3-jährige obere Se- } \\
\text { kundarschulbildung } \\
\text { Zuständigkeit: } \\
\text { Bundesstaaten }\end{array}$ & $\begin{array}{l}\text { Berufsbildung } \\
\text { (Educaçáo Profissional): } \\
\text { 1. Berufliche Grundbildung } \\
\text { (nivel básico) } \\
\text { 2. Technisches Niveau } \\
\text { (nivel técnico), } \\
\text { 3. Technologisches Niveau } \\
\text { (nivel tecnólogo), } \\
\text { berechtigt zur Bewerbung an } \\
\text { Hochschulen } \\
\text { Zuständigkeit: } \\
\text { Bundesstaaten und mehrheitlich } \\
\text { privatwirtschaftliche Bildungsin- } \\
\text { stitutionen, die den allgemeinen } \\
\text { Bildungsauftrag nach den staatli- } \\
\text { chen Richtlinien wahrnehmen. }\end{array}$ \\
\hline \multirow{3}{*}{ ab 18} & \multirow{3}{*}{$\begin{array}{l}\text { Höhere Bildung } \\
\text { (Ensino Superior) } \\
\text { Zuständigkeit: } \\
\text { Bundesregierung, } \\
\text { Bundesstaaten, } \\
\text { Gemeinden }\end{array}$} & \multicolumn{2}{|c|}{$\begin{array}{l}\text { Grundständiges Studium (Graduaçáo) } \\
\text { mit Abschluss Bacharel oder Licenciatura }\end{array}$} \\
\hline & & \multicolumn{2}{|l|}{ Masterstudium } \\
\hline & & \multicolumn{2}{|l|}{ Promotion } \\
\hline
\end{tabular}

Quelle: Eigene Zusammenstellung 
von theoretischem Wissen mit der praktischen Anwendung gefördert werden. Ein Abschluss der mittleren Bildung ist die Voraussetzung für den Übergang zur höheren Bildung.

Die höhere Bildung (Ensino Superior) ist so gestaltet, dass ein erster akademischer Grad nach etwa vier bis fünf Jahren im Rahmen der Graduação mit einem Bachelor (Bachare) erreicht werden kann. Parallel hierzu existiert das berufsqualifizierende Studium, welches zu einer Lehrertätigkeit berechtigt. Nach ebenfalls vier bis fünf Jahren wird hierbei der Titel Licenciatura verliehen. Beide Abschlüsse berechtigen zu einem Postgraduiertenstudium. Dieses endet entweder nach einem in der Regel zweijährigen Masterstudium oder einer daran anschließenden Promotion. Zusätzlich besteht die Möglichkeit des Besuchs von zweijährigen Fortbildungskursen (Cursos Sequenciais). Diese berechtigen allerdings nicht zur Aufnahme eines Postgraduiertenstudiums (Richter 2013: 183). Die verschiedenen Hochschulen bieten neben ihrem regulären Präsenzprogramm zusätzlich seit dem Jahr 2000 Fernstudiengänge an. Etwa $4 \%$ der angebotenen Studiengänge können inzwischen als Fernstudium absolviert werden (BMBF 2012).

Nach der Fundamentalbildung kann auch der Weg der Berufsbildung (Educação Profissional) eingeschlagen werden. Sie unterteilt sich in drei Stufen: die berufliche Grundbildung (nivel básico), das technische Niveau (nivel técnico) und das technologische Niveau (nivel tecnólogo). Die berufliche Grundbildung ermöglicht erste Berufsbefähigungen und "dient [...] der Qualifikation von Arbeitskräften bzw. der Ermöglichung des Wiedereintritts in das Berufsleben, unabhängig von irgendwelcher Vorbildung" (Fritsche 2003: 126). Im Rahmen des technischen Niveaus erhalten die Schüler eine Ausbildung, die einer Berufsausbildung entspricht. Der größte "Teil der Berufsbildung in Brasilien findet [...] außerhalb des staatlichen Bildungssystems statt, und zwar in sektorbezogenen, dezentral organisierten, privatwirtschaftlichen Berufsbildungsinstitutionen, die privatwirtschaftlich finanziert werden und den Arbeitgeberverbänden unterstehen" (Richter 2013: 184). Das Bildungsgesetz sieht dabei vor, dass diese Berufsschulen ebenfalls den allgemeinen Bildungsauftrag wahrnehmen und den Berufsschülern einen Abschluss der mittleren Bildung ermöglichen. Ebenfalls ist es möglich, den Grad der mittleren Bildung parallel zur Berufsausbildung in speziellen Kursen an einer öffentlichen Mittelschule zu erlangen. Auf dem technischen Niveau aufbauend, bieten weiterführende berufsbildende Schulen eine theoretisch-technisch orientierte Ausbildung an, im Rahmen derer das technologische Niveau mit dem Diploma 
de Tecnólogo absolviert werden kann. Dieser Abschluss berechtigt ebenfalls zur Bewerbung an einer Hochschule (Fritsche 2003: 126; Bezerra Andrade 2005: 31; Richter 2013: 184).

\section{Die Grundbildung}

Als Ergebnis der Bildungs- und Sozialpolitik der Regierungen Cardoso (1995-2002) und Lula da Silva (2003-2011) lassen sich heute einige Fortschritte des brasilianischen Bildungswesens und des allgemeinen Bildungsstands der Bevölkerung erkennen. Wichtige politische Grundlagen für diese Entwicklungen waren die Einrichtung der Bildungsfonds FUNDEF/FUNDEB, die Implementierung der an Konditionen gebundenen Bildungs- und Sozialhilfeprogramme Bolsa Escola und Bolsa Família und die Evaluierung von Schülerleistungen durch nationale (SAEB/ProvaBrasil) und internationale (PISA) Erhebungen.

Mit FUNDEF (Fundo de Desenvolvimento do Ensino Fundamental), dem Bildungsfond zur Entwicklung der Fundamentalbildung, wurde eine landesweite Verbesserung der Grund- und unteren Sekundarschulbildung (Schuljahr 1 bis 9) angestrebt. Das Hauptaugenmerk des Programms lag auf der garantierten Bereitstellung eines Mindestlevels an Bildungsausgaben pro Schüler, welches im Laufe der Zeit zusätzlich erhöht wurde. Es wurde festgelegt, dass $60 \%$ der Ausgaben für die Bezahlung von Lehrern und $40 \%$ für weitere Kosten von Schulen verwendet werden müssen. Mit FUNDEF bezog sich die Bildungspolitik der Regierung Cardoso insbesondere auf die Problematik der ungleichen Finanzierung des Bildungswesens zwischen den verschiedenen Regionen des Landes. Das dezentrale Bildungssystem sieht vor, dass sich die Staaten und Kommunen die Verantwortung für das Bildungsangebot teilen. Die Kommunen und Städte sind für die Bereitstellung und Verwaltung vorschulischer Erziehungs- und Bildungseinrichtungen sowie für einen Teil der Grundschulen zuständig. Während sich die Bildungssituation der reicheren Bundesstaaten weniger problematisch gestaltete, waren insbesondere die Kommunen der zentralwestlichen, nordöstlichen und nördlichen Bundesstaaten von massiver Unterfinanzierung betroffen. Hier fehlte es an Grundlegendem: Lehrbücher und Einrichtungsgenstände, die Versorgung der Schulgebäude mit Elektrizität und Wasser sowie eine hinreichende Ausbildung und Bezahlung von Lehrkräften konnte nicht in allen Regionen Brasiliens gewährleistet 
werden. Die mit FUNDEF verbundenen Bestimmungen verpflichteten die Bundesstaaten dazu, finanzielle Mittel so aufzuteilen, dass sowohl bundesstaatliche als auch kommunale Bildungseinrichtungen das gesetzliche Minimum an Finanzierung erhalten. Die Staaten, welche auf Grund zu geringer Steuereinnahmen nicht in der Lage waren, für alle Schulen dieses Minimum aufzubringen, wurden durch den Bildungsfond bezuschusst. Im Schnitt wurden hierbei jährlich 6 von 26 Bundesstaatshaushalten durch den Zentralhaushalt aufgestockt. Durch seine enorme ressourcenerhöhende Wirkung stellte FUNDEF insbesondere in den Kommunen dieser Regionen ein Anreizsystem zur Erhöhung der Schülerzahlen dar. Seit 1989 können verstärkte Bemühung der Kommunen zur Erhöhung der Einschulungszahlen in Grundschulen beobachtet werden (z. B. in Form von Schulbustransport, Schulessen und Einschulungskampagnen). Im Jahr 2007 wurde unter der Administration von Lula da Silva die Fortsetzung und Ausweitung des Programms beschlossen: aus FUNDEF wurde FUNDEB (Fundo de Manutenção e Desenvolvimento da Educação Básica e de Valorização dos Profissionais da Educação). In das Bildungsfinanzierungsprogramm wurden nun auch die vorschulische Bildung, die höhere Sekundarstufe (Klasse 10 bis 12) und die Erwachsenenbildung mit einbezogen. Außerdem garantiert FUNDEB eine Pro-Kopf-Finanzierung für die Einschulungen Angehöriger der indigenen Bevölkerung und der Bewohner der Quilombolas (Bruns/Evans/Luque 2012: 1-6).

Im Zuge dieser kontinuierlichen Bildungsfinanzierungspolitik wurden erhebliche Verbesserungen bezüglich der Einschulungsraten erreicht. Während im Jahr 2005 lediglich 83 \% der Sechsjährigen eine Grundschule besuchten, stieg diese Zahl bis 2010 auf $92 \%$ an. Auch im Bereich der vorschulischen Bildung lässt sich ein Anstieg verzeichnen. Der Anteil der Dreijährigen, die eine vorschulische Einrichtung besuchten, stieg im Zeitraum von 2005 bis 2010 von $21 \%$ auf $32 \%$ an, bei den Vierjährigen ließ sich ein Anstieg von $37 \%$ auf $55 \%$ verzeichnen und bei den Fünfjährigen stieg der Anteil von $63 \%$ auf $78 \%$ an (OECD 2012). Als weiterer Indikator für den positiven Effekt des Bildungsfonds können die stark gestiegenen Bildungsausgaben Brasiliens herangezogen werden. Den Daten der OECD zur Folge stieg der Anteil der staatlichen Gesamtausgaben für Bildung (hierin sind Ausgaben für Bildungseinrichtungen, Verwaltungen und Subventionen für private Einrichtungen einbezogen) in den Jahren 2000 bis 2009 von $10,5 \%$ auf 16,8\%. Verglichen mit den vorliegenden Daten anderer Länder weist Brasilien hiermit die stärkste Ausgabenanstiegskurve 
auf und lag im Jahr 2009 sogar über dem OECD-Durchschnitt von $13 \%$ (OECD 2012; Bruns/Evans/Luque 2012: 6-7).

Im Jahr 2001 wurde das Schulstipendienprogramm Bolsa Escolar ins Leben gerufen, welches im Jahr 2003 in das umfassende Sozialhilfeprogramm Bolsa Familia integriert wurde. Es handelt sich um Programme, mit deren Hilfe allen Kindern die Möglichkeit gegeben werden soll, Schulen im Rahmen der Fundamentalbildung zu besuchen und abzuschließen. Einkommensschwache Familien mit Kindern im schulpflichtigen Alter erhalten einen monatlichen Pro-Kopf-Zuschuss ausgezahlt, wenn die Kinder eine öffentliche Schule besuchen (Bruns/Evans/Luque 2012: 8-11). Studienergebnisse zeigen, dass diese conditional cash-transfer-Programme einen Beitrag zur Erhöhung der Anwesenheitszeiten der Schüler in Schulen und zur Verringerung der Schulabbrecherquote leisten. So war im Jahr 2007 die Zahl unentschuldigt fehlender Kinder aus armen Familien, welche finanziell unterstützt wurden, um 3,6\% geringer als die der Kinder, deren Familien nicht unterstützt wurden. Auch die Wahrscheinlichkeit des Schulabbruchs war bei Kindern von unterstützten Familien um 1,6 \% geringer als bei Kindern, deren Familien keine Unterstützung erhielten (Veras Soares/Perez Riba/Guerreiro Osório 2007: 5).

Bis 1994 existierte in Brasilien keine Möglichkeit zur Erfassung von Schülerlernerfolgen. Unter den Regierungen Cardoso und Lula da Silva wurden systematisch Verfahren zur Messung von Lernleistungen entwickelt und implementiert. Im Jahr 1995 wurde das Evaluierungsverfahren SAEB (Sistema Nacional de Avaliação da Educação Básica) eingeführt, im Rahmen dessen im halbjährlichen Rhythmus die Leistungen einer Stichprobe von Schülern der 4., 8. und 11. Klasse in den Fächern Mathematik und Portugiesisch getestet werden. Im Jahr 2005 wurde das Evaluierungsprogramm durch Prova Brasil auf alle Schüler der 4. und 8. Klasse ausgeweitet, welches nun alle zwei Jahre die nationalen Schülerleistungen erfasst. Mit SEAB werden weiterhin anhand einer Stichprobe die Lernerfolge von Elftklässlern erhoben. Darüber hinaus nimmt Brasilien seit 2000 an den internationalen Schülerleistungsevaluationen der OECD im Rahmen der PISA-Studie (Programme for International Student Assessment) teil. Hierbei werden im dreijährigen Rhythmus die Lernerfolge der Sekundarschulabsolventen (9. Klasse) evaluiert (Bruns/Evans/Luque 2012: 8).

Im Jahr 2000 landeten Brasiliens Schüler in den drei durch PISA evaluierten Bereichen Mathematik, Naturwissenschaften und Lesekompetenz jeweils auf dem letzten Platz von 32 teilnehmenden Ländern. Die Erhe- 
bung von 2009 zeigt, dass Brasilien einige Verbesserungen vorzuweisen hat. So erreichten die Schüler in den drei Disziplinen im Schnitt 33 Punkte mehr als im Jahr 2000, was im Vergleich die drittgrößte Verbesserung innerhalb dieser Periode bedeutet. In Mathematik erzielten Brasiliens Schüler mit einem Anstieg von 52 Punkten im Vergleich mit den in der gleichen Periode teilnehmenden Ländern die größte Verbesserung (Bruns/Evans/ Luque 2012: 16).

Die beschriebenen Fortschritte dürfen jedoch nicht darüber hinweg täuschen, dass die brasilianische Bildungspolitik das traditionelle Problem der regionalen Ungleichheiten noch nicht zu lösen vermochte. Zwar haben inzwischen größere Teile der brasilianischen Bevölkerung Zugang zum Bildungssystem und im Schnitt verbleiben sie auch länger in diesem; im Nordosten beträgt der Anteil derjenigen, die weniger als vier Jahre zur Schule gehen, jedoch noch immer $40 \%$, während der Landesdurchschnitt bei $26 \%$ liegt (BMBF 2012). Die Inklusion der Bevölkerung in das Bildungssystem - insbesondere in den ärmeren Regionen - stellt somit weiterhin eine wichtige Herausforderung dar. Darüber hinaus zeigt sich, dass trotz quantitativer Verbesserungen, die Qualität der Schulbildung nach wie vor erheblichen Verbesserungsbedarf aufweist, denn Schüler lernen vergleichsweise wenig in brasilianischen Schulen. Auch wenn sich in der PISA-Studie 2009 Verbesserungen abzeichneten, so erreichten im Bereich Mathematik $38 \%$ der getesteten Schüler nicht einmal die niedrigste Kompetenzstufe 1 (auf einer Skala von 1 bis 6), was bedeutet, dass ein sehr großer Teil der brasilianischen Schüler im Alter von 15 Jahren nicht über die notwendigen grundlegenden Kenntnisse und Fähigkeiten verfügt, um mathematische Basisoperationen durchzuführen (OECD 2010: 130-131). Darüber hinaus hat sich die beschriebene Verbesserung lediglich im untersten Leistungsbereich vollzogen. Der Anteil derjenigen Schüler, die ein sehr hohes Ergebnis im Bereich Mathematik erreichten, ist mit $1 \%$ nach wie vor sehr gering. Brasilien liegt mit diesem Ergebnis weit unter dem OECD-Durschnitt von $15 \%$. Im Bereich der Lesekompetenz lässt sich ein noch weniger ermutigendes Bild erkennen: Während nur 1,3\% der getesteten Schüler und Schülerinnen das höchste Kompetenzniveau erreichten, gelangten $50 \%$ von ihnen in ihren Testergebnissen nicht über die niedrigste Kompetenzstufe 1 hinaus (Bruns/Evans/Luque 2012: 22-25). Dies bedeutet, dass die Hälfte der 15-jährigen maximal über elementare Lesefähigkeiten verfügt, mit deren Hilfe sie lediglich explizite Informationen und Hauptaussagen aus einem Text mit vertrauten Inhal- 
ten herausarbeiten und mit bekannten Alltagsthemen verbinden können (OECD 2010: 52).

Des Weiteren schafft es ein großer Teil der brasilianischen Schüler nicht, Schulabschlüsse innerhalb der vorgesehen Zeit zu erreichen. Weltweit weist Brasilien eine der höchsten Wiederholungsraten von Schülern im Grund- und Mittelschulbereich auf. Nicht selten kommt es vor, dass Schüler für das Abschließen der neunjährigen Fundamentalbildung drei Schuljahre mehr benötigen. Diese Ineffektivität der Schulbildung bedeutet nicht nur für die Schüler und ihre Familien einen beachtlichen Mehraufwand an Ressourcen, auch auf die staatlichen Ausgaben für Bildung hat dies erhebliche Auswirkungen: Mehr als 12\% der Gesamtbildungsausgaben im Bereich der Fundamentalbildung werden für Schüler aufgewendet, welche Klassen wiederholen müssen (Bruns/Evans/Luque 2012: 48). Im engen Zusammenhang mit der hohen Wiederholungsquote steht die hohe Schulabbrecherquote. Obwohl in allen Regionen Brasiliens die Einschulungsraten für Grundschulen bei mindestens $90 \%$ liegen, ist für das Jahr 2010 noch immer festzustellen, dass ein nicht unerheblicher Teil der Schüler keinen Abschluss der Fundamentalstufe erreichte. Hiervon sind besonders Schüler in ländlichen Regionen und Schüler aus Familien mit sehr geringen Einkommen betroffen. Im Jahr 2009 konnten in ländlichen Regionen aus der Gruppe der 26-30jährigen lediglich $35 \%$ einen Abschluss der Fundamentalbildung nachweisen. In urbanen Regionen haben $75 \%$ dieser Alterskohorte diesen Abschluss erreicht. Landesweit verlassen etwa $30 \%$ der Schüler die Schule nach 11 bis 12 Jahren, ohne einen Abschluss der Fundamentalstufe erworben zu haben. Im Mittelschulbereich brechen ca. $25 \%$ der Schüler die Schule ab, nachdem es ihnen nach vier Jahren Schulbesuch nicht gelungen ist, die mittlere Reife zu erlangen. Mehr als $15 \%$ der Mittelschulabsolventen haben bereits das 25. Lebensjahr vollendet (Bruns/Evans/Luque 2012: 32-34, 47).

Die beschriebenen Probleme bedingen und verstärken sich durch einen Mangel an gut ausgebildeten Lehrern an öffentlichen Schulen. Viele Lehrer verfügen nicht über einen Hochschulabschluss. Dieser Umstand lässt sich damit erklären, dass bis in die 1990er Jahre ein Hochschulabschluss keine Voraussetzung für eine Lehrertätigkeit war. Zusätzlich erklärt dies, warum der Lehrerberuf bis heute wenig öffentliches Ansehen genießt (Richter 2013: 188). Erst mit dem im Jahr 1996 erlassenen Bildungsgesetz wurde die Ausbildung von Lehrern auch zur Aufgabe der Hochschulen erhoben. Ein Hochschulabschluss ist jedoch bis heute nur 
für Sekundarschullehrer, nicht aber für Grundschullehrer notwendig. Die Ausbildung von Vor- und Grundschullehrern erfolgt vorwiegend in speziellen Kursen in den Sekundarschulen (Richter 2013: 185). Die schlechte Bezahlung und die geringen Aufstiegschancen von Lehrern an öffentlichen Schulen lassen den Beruf wenig attraktiv erscheinen. Die Einstiegsgehälter übersteigen häufig nicht das Zweifache des gesetzlichen Mindestlohns, sodass viele Lehrer - insbesondere bei einer Teilzeitbeschäftigung oder einer Beschäftigung auf Stundenbasis - an mehreren Schulen lehren, um sich ein ausreichendes Monatsgehalt zu verdienen. Die geringste Entlohnung erhalten Lehrer in der Regel an kommunalen Schulen. Hierzu kommen die schlechten Arbeitsbedingungen, denen Lehrer auf Grund überfüllter Schulklassen und mangelhafter Ausstattung der Schulen ausgesetzt sind. Im Durchschnitt unterrichtete im Jahr 2010 ein Grundschullehrer 23,4 Schüler (OECD-Durchschnitt: 15,8 Schüler) und ein Mittelschullehrer 19 Schüler (OECD-Durchschnitt: 13,8). Im selben Jahr betrug die durchschnittliche Klassengröße in Grundschulen 24,6 Schüler (OECD-Durchschnitt: 21,2) und in Sekundarschulen 29 Schüler (OECDDurchschnitt: 23,4 Schüler). Viele Lehramtsstudenten nutzen die freien Studienplätze an öffentlichen Universitäten lediglich, um einen ersten akademischen Abschluss zu erwerben. Nicht selten werden sie anschließend jedoch in einer anderen Branche tätig oder bemühen sich um eine Lehrtätigkeit in einer privaten Schule, die ihnen eine höhere Bezahlung und bessere Arbeitsbedingungen bietet (Hamm 2011: 2).

Eine der wichtigsten Herausforderungen der Bildungspolitik wird es daher sein, die Qualität der schulischen Lehre zu verbessern. Hierbei müssen zum einen Anreize geschaffen werden, die die Attraktivität des Lehrerberufs erhöhen. Zum anderen muss an der Verbesserung der Ausund Weiterbildung von Lehrern gearbeitet werden, denn häufig wissen auch engagierte Lehrer schlichtweg nicht, wie sie ihren Unterricht verbessern können (Bruns/Evans/Luque 2012: 64), was sich wiederum negativ auf ihre Motivation und auch das Ansehen der Lehrer auswirkt.

Aspekte des inhaltlichen und pädagogisch-methodischen Wissens könnten daran anschließend in Rekrutierungsverfahren für den Lehrerberuf aufgegriffen werden. Zwar sind die Einstellungsverfahren für Lehrer in Brasilien dezentral organisiert, sodass hier kein einheitliches Bild präsentiert werden kann. Es lässt sich jedoch beobachten, dass viele Eignungs- und Bewerbungsverfahren eine schriftliche Klausur und die Bewertung formaler Abschlüsse beinhalten. Anhand der erreichten Ergebnisse 
werden Rangfolgen erstellt, auf deren Grundlagen die Entscheidung für eine Einstellung der Bewerber getroffen wird. Aufgaben wie das Erstellen eines Unterrichtsplans oder das Abhalten einer beobachteten Unterrichtsstunde müssen in der Regel von den Bewerbern nicht erbracht werden. Es zeigt sich jedoch, dass Länder mit einem hohen Bildungsstand und guten Schülerleistungen in der Tendenz strengere Auswahlverfahren für die Einstellung neuer Lehrer vorsehen (Bruns/Evans/Luque 2012: 58).

Angesichts der Probleme des öffentlichen Schulsystems versuchen viele Familien mit höherem Einkommen, ihren Kindern den Besuch einer privaten Schule zu ermöglichen. Dies kann sich jedoch nur ein kleiner Teil der brasilianischen Bevölkerung leisten. Der Anteil aller Mittelschulschüler, welche eine öffentliche Einrichtung besuchen, beträgt $78 \%$. Das Einkommen ihrer Familien beträgt in etwa ein Drittel des Familieneinkommens der Schüler an privaten Mittelschulen (Schwartzman 2013). Hierin zeigt sich, dass der Zugang zu guter Bildung noch immer sehr vom Einkommen der Eltern abhängt.

Eine häufige Forderung zur Verbesserung des Bildungswesens ist der Ruf nach mehr öffentlichen Ausgaben für Bildung. Das brasilianische Bildungsministerium reagierte darauf, indem es im Herbst 2012 mit der Verabschiedung des Nationalen Bildungsplans festlegte, dass der Ausgabenanteil für Bildung bis zum Jahr 2020 auf $10 \%$ des Bruttoinlandprodukts angehoben werden soll (The Economist: 28.10.2012). Dieses ambitionierte Ziel zeigt, dass das Bildungswesen und seine Finanzierung auch in der kommenden Dekade eine wichtige Rolle in der brasilianischen Politik spielen werden. Ob eine derartige Erhöhung der Ausgaben erfolgversprechend ist, kann jedoch bezweifelt werden. Bruns et al. (2012) befürchten, dass eine Erhöhung des Ausgabenanteils durch kurzfristige Investitionen zur Verschwendung öffentlicher Mittel führen könnte. Hierfür sprechen zum einen Vergleiche mit anderen Ländern, bei denen bisher kein bedeutender Zusammenhang zwischen einer Erhöhung des Ausgabenanteils für Bildung und einer Verbesserung der Schülerleistungen festgestellt werden konnte. Zum anderen ist zu beobachten, dass das Bruttoinlandsprodukt Brasiliens einen starken Zuwachs bei einem rückgängigen Bevölkerungswachstum erlebt. Es ist abzusehen, dass die Zahl der Schüler - insbesondere die der Grundschüler - in den kommenden Jahren zurückgehen wird, sodass sich der Ausgabenanteil gemessen am Bruttoinlandsprodukt pro Schüler erhöhen wird, ohne dass eine Erhöhung des Ausgabenanteils vorgenommen werden muss. Daher schlagen Bruns et. al vor, dass Brasili- 
en dem Beispiel anderer Länder folgen sollte, die in der Vergangenheit vor dieser Situation standen, indem die durch schrumpfende Schülerzahlen freigesetzten Ressourcen in die Verbesserung der Qualität des Bildungswesens investiert werden. Frei werdende Infrastruktur, die mit zurückgehenden Grundschülerzahlen zu erwarten ist, könnte für die Sekundarschulbildung genutzt werden. Darüber hinaus könnten freie Ressourcen in die Verbesserung und Ausweitung der vorschulischen Bildung investiert werden, um zu gewährleisten, dass Kinder mit der Einschulung angemessen darauf vorbereitet sind, im Rahmen des Schulunterrichts zu lernen (Bruns/Evans/Luque 2012: 41-44).

\section{Die höhere Bildung}

Seit den 1990er Jahren erhält die höhere Bildung zunehmend gesellschaftliche Beachtung. Mit wachsender Nachfrage nach qualifizierten Arbeitskräften und einer steigenden Zahl von Mittelschulabsolventen "mündete das elitäre Bild der Hochschulbildung in eine allgemeine Auffassung von Hochschulbildung als Berufsausbildung einer qualifizierten Erwerbsbevölkerung" (Balbachevsky/Schwartzman 2010: 612). Im Zuge dessen hat sich das Angebot im Bereich der höheren Bildung stark vergrößert. Dies äußert sich zum einen in einer Ausweitung des Spektrums der angebotenen Studiengänge, zum anderen kam es seit Ende der 1990er Jahre zu einer Neugründungswelle entsprechender Organisationen. Im Zeitraum von 1998 bis 2008 hat sich die Zahl der vorhandenen Hochschulen fast verdoppelt, was vor allem auf eine Vergrößerung des Hochschulangebots des privaten Sektors zurückführen ist. Insgesamt waren im Jahr 2008 rund $90 \%$ aller brasilianischen Hochschulen privat finanzierte Bildungseinrichtungen (Dias et al.: 2011: 1). Seit dem Jahr 1999 hat sich die Zahl der in privaten Hochschulen eingeschriebenen Studierenden mehr als verdoppelt. Insgesamt waren im Jahr 2011 etwa $75 \%$ aller Studierenden in privaten Einrichtungen registriert (BMBF: 2012). Die öffentlichen Hochschulen (10\%) sind zu etwa gleichen Teilen in der Trägerschaft der Zentralregierung, der Bundesstaaten und der Gemeinden (Balbachevsky/Schwartzman 2010: 602).

Mit der Expansion der Hochschulbildung ist zudem eine Diversifizierung der verschiedenen Einrichtungen bezüglich des Studienfachangebots, der Organisationsform und der Qualität von Lehre und Forschung zu beobachten. So gelten die großen, fast ausschließlich staatlich finan- 
zierten Universitäten mit ihrem breiten Angebot an Graduierten- und Postgradiertenstudiengängen nicht nur als gute und günstige Lehreinrichtungen, sondern bieten mit ihren Forschungszentren auch nationalen und internationalen Wissenschaftlern attraktive Arbeitsbedingungen (Fritsche 2003: 66). Sie repräsentieren etwa die Hälfte aller Organisationen mit Universitätsstatus und bilden mehr als $80 \%$ der Studierenden in postgraduierten Programmen aus (Balbachevsky/Schwartzman 2010: 602). Innerhalb der Gruppe der kleineren, häufig privaten Bildungseinrichtungen variiert das Ausmaß angebotener Studiengänge stark. Es existieren beispielsweise stark spezialisierte Organisationen, die sich nur auf einen Fachbereich konzentrieren und somit nur entsprechend wenige Studiengänge anbieten. Diese Organisationen nennen sich nicht Universitäten, sondern Fakultäten. Viele private Hochschulen funktionieren vorwiegend als reine Lehreinrichtungen für grundständige Studiengänge, ohne darüber hinaus Postgraduiertenstudiengänge anzubieten und einen Forschungsbereich aufzuweisen (Müller 2009: 12).

Zwar kann nicht pauschal behauptet werden, dass die Qualität der Ausbildung in privaten Hochschulen geringer ist als in den öffentlichen Hochschulen, jedoch fehlt es bisher an zuverlässigen Evaluierungsinstrumenten. Unter der Regierung Cardoso wurde zwar ein Evaluierungsverfahren zur Beurteilung der Qualität grundständiger Studiengänge eingeführt; hierfür wurden Ranglisten zur Bewertung von Studiengängen veröffentlicht. Zu ihrer Erstellung bezog sich das Bildungsministerium auf die infrastrukturelle Ausstattung der Hochschulen, auf Informationen zum akademischen Grad der Lehrenden und auf Leistungstestergebnisse aller Studierenden der jeweiligen Ausbildungsrichtung. Dieses Verfahren wurde jedoch unter Lula da Silva durch das Verfahren ENADE (Exame Nacional de Desempenho dos Estudantes) abgelöst. Hierbei werden die Lernleistungen der Studenten nur noch anhand einer Stichprobe erhoben. Dieses Evaluierungsverfahren "hat erhebliche methodische Schwächen; die Regierung selbst sagt[e], dass die Ergebnisse nicht für bare Münze genommen werden sollten" (Balbachevsky/Schwartzman 2010: 616). Insgesamt haben sich in einigen Bundesstaaten und Gemeinden im privaten Sektor sehr schnell neue Studienprogramme und Organisationen gegründet, die noch keinen regulierenden Prozessen und Qualitätsüberprüfungen unterzogen werden konnten (Dias et al. 2011: 17).

Für die Aufnahme an einer öffentlichen Hochschule spielt die Durchschnittsnote des mittleren Bildungsabschlusses keine große Rolle. Er be- 
rechtigt lediglich zur Teilnahme an den Aufnahmeprüfungen - dem Vestibular - der öffentlichen Hochschulen. Diese wird von den einzelnen Einrichtungen individuell gestaltet und beinhaltet neben Prüfungsinhalten bezüglich des angestrebten Studiengangs auch Wissenstests zu allen Grundfächern der Schulbildung. Die Hochschulen erteilen Zulassungen nach Reihenfolge der erreichten Testergebnisse - bis ihre Kapazitäten erschöpft sind (Waber 2007: 44). In der Regel sind Absolventen privater Mittelschulen wesentlich besser auf das Vestibular vorbereitet, während Absolventen öffentlicher Schulen häufig das notwendige Wissen fehlt, um ein Ergebnis in den vorderen Rängen zu erreichen. Um ihre Zulassungschancen zu erhöhen und die Kosten für die Teilnahme an den Zulassungsklausuren nicht umsonst zu investieren, nehmen viele Mittelschulabsolventen an speziellen ein- bis zweijährigen Kursen teil, die auf das Vestibular vorbereiten. Einige Schulen, vor allem des privaten Sektors, haben diese Vorbereitungskurse bereits in ihren Unterricht integriert. Im Normalfall handelt es sich jedoch um kostenpflichtige Schulungen privater Anbieter, die nicht selten damit werben, Hochschullehrer zu beschäftigen, die schon einmal an der Gestaltung von Verstibularklausuren mitgewirkt haben (Fritsche 2003: 6-7).

Viele Familien, deren Kinder an öffentlichen Hochschulen keinen Studienplatz erhalten haben, versuchen ihnen zumindest an einer günstigeren privaten Hochschule ein Studium zu ermöglichen. Die Zahl der Einschreibungen in private Hochschulen ist in den vergangen Jahren stark angewachsen: Im Jahr 2008 waren 74,9\% der brasilianischen Studierenden in private Hochschulen eingeschrieben. Gleichzeitig blieben jedoch im selben Jahr 40 \% der vorhandenen Gesamtstudienplätze, die fast ausschließlich dem privaten Sektor zugeordnet waren, unbesetzt. Dies zeigt, dass viele Mittelschulabsolventen Schwierigkeiten haben, die Kosten für ein privates Hochschulstudium aufzubringen (Dias et al. 2011: 11-12). Um mehr Absolventen öffentlicher Mittelschulen eine Hochschulausbildung zu ermöglichen, hat die Regierung Lula da Silva im Jahr 2004 das Programm 'Universität für alle' (Programa Universidade para Todos) auf den Weg gebracht. Mit diesem Programm werden in privaten Einrichtungen "quasi öffentliche Studienplätze geschaffen" (BMBF 2012), indem private Hochschulen finanziell bezuschusst werden, wenn sie einkommensschwachen oder einer Minderheit angehöhrenden Studierenden die Studiengebühren erlassen (Balbachevsky/Schwartzman 2010: 616). Von 2005 bis 2011 wurden fast 750.000 Studierende durch das Programm gefördert, davon knapp $70 \%$ durch Vollstipendien (BMBF 2012). 
Zur Förderung öffentlicher Hochschulen wurde im Jahr 2008 das Programm Reuni (Programa de Apoio a Planos de Reestruturação e Expansão das Universidades Federais) auf den Weg gebracht. Hierbei werden finanzielle Mittel zur Gründung neuer Hochschulstandorte und für interne Umstrukturierungen vorhandener Hochschulen zur Verfügung gestellt, mit dem Ziel, mehr öffentliche Studienplätze zu schaffen (BMBF 2012). Des Weiteren unterschrieb die amtierende Präsidentin Dilma Rousseff im Sommer 2012 ein neues Hochschulgesetz, welches öffentliche Universitäten verpflichtet, bis zum Jahr 2016 die Hälfte der verfügbaren Studienplätze für Absolventen öffentlicher Schulen zur Verfügung zu stellen (Schwartzman 2013). Die Erfolge der jüngsten Beschlüsse bleiben noch abzuwarten. Um eine Erhöhung der Akademikerrate zu erreichen, muss die Bildungspolitik unweigerlich ihren Schwerpunkt weiterhin auf die Verbesserung der Grundbildung - und hier insbesondere des Sekundarschulbereichs - richten. Denn auch wenn nun mehr Mittelschulabsolventen ein Hochschulstudium anstreben, so zeigt sich auch für die Hochschulausbildung eine große Diskrepanz zwischen den Einschreibzahlen und der Anzahl derjenigen, die schließlich einen Hochschulabschluss erwerben. Von denjenigen, die sich in den Jahren 1991 bis 2008 in eine Hochschule eingeschrieben haben, erreichten im Durchschnitt lediglich $50 \%$ einen Abschluss (Dias et al. 2011: 15). Ein möglicher Grund hierfür könnte sein, dass den Studenten die finanziellen Mittel für das Beenden ihres Studiums fehlen. Dem könnte durch die Quotenreglung Abhilfe geschaffen werden. Jedoch dürfte insbesondere auch fehlendes Wissen, verschuldet durch schlechte Vorbereitung der Studierenden durch die Mittelschulen, ein entscheidender Grund für das Abbrechen eines Hochschulstudiums sein. Daher besteht die Befürchtung (Schwartzman 2013), dass die Hochschulen auf ihre neue Klientel mit einer Herabsetzung der Leistungsanforderungen reagieren. Dies könnte sich negativ auf die Qualität der öffentlichen Hochschullehre auswirken.

\section{Fazit}

Das brasilianische Bildungssystem hat seit den 1990er Jahren große Fortschritte gemacht. Sowohl die Grundbildung als auch die höhere Bildung haben sich in quantitativer und qualitativer Hinsicht positiv entwickelt. Gleichwohl schlagen sich die gravierenden sozialen und regionalen Dis- 
paritäten bis heute auch im Bildungssystem nieder. Um dauerhaft der Herausforderung gewachsen zu sein, der Bevölkerung Aufstiegschancen zu bieten, für mehr soziale Gerechtigkeit zu sorgen und gleichzeitig die Grundlagen für eine zukunftsfähige Entwicklung des Landes weiter zu verbessern, muss vor allem die Qualität der öffentlichen Schulbildung weiter verbessert werden.

\section{Literaturverzeichnis}

Balbachevsky, Elizabeth/Schwartzman, Simon (2010): "Das Hochschulsystem. Strukturen und Veränderungstendenzen”. In: Costa, Sérgio/Kohlhepp, Gerd/Nitschack, Horst/Sangmeister, Hartmut (Hg.): Brasilien heute. Geographischer Raum, Politik, Wirtschaft, Kultur. 2. vollständig neu bearbeite Auflage. Frankfurt am Main: Vervuert, 601-622.

Bezerra Andrade, Francisca R. (2005): "Die Entwicklung der mittleren und beruflichen Bildung in Brasilien". In: Berufsbildung in Wissenschaft und Praxis (BWP), 1, S. 30-34. Verfügbar unter: < http://www.bibb.de/veroeffentlichungen/de/publication/show/ id/1686> (14.06.2013).

BMBF (2012): Bildung und Forschung: Brasilien. Bundesministerium für Bildung und Forschung, Kooperation International. Verfügbar unter: <http://www.kooperation-international.de/buf/brasilien.html> (14.06.2013).

Bruns, Barbara/Evans, David/Luque, Javier (2012): Achieving World-Class Education in Brazil. The Next Agenda. Washington: The World Bank.

Da Trindade Prestes, Emília Maria/Pfeiffer, Dietmar K. (2010): “Überwindung der Bildungsarmut durch staatliche Bildungspolitik: Möglichkeiten und Grenzen”. In: Sandkötter, Stephan (Hg.): Bildungsarmut in Deutscbland und Brasilien. Frankfurt am Main.u. a: Peter Lang, 39-68.

Dias, Diana/Marinho-Araújo, Claisy/Almeida, Leandro/Amaral, Alberto (2011): The Democratisation of Access and Success in the Higher Education: The Case of Portugal and Brazil. Higher Education Management and Policy, Vol. 32/1. Verfügbar unter: <http:// www.oecd-ilibrary.org/education/the-democratisation-of-access-and-success-inhigher-education_hemp-23-5kgglbdlrptg> (14.06.2013).

FRITSCHE, Michael (2003): Bildungspolitik und wirtschaftliches Wachstum in Brasilien. Marburg: Tectum Verlag.

НАмм, Esther (2011): Die brasilianische Bildungsmisere - Hindernis für den sozialen Aufstieg. Online-Publikation der Konrad-Adenauer-Stiftung. Verfügbar unter: <http://www.kas. de/wf/doc/kas_23172-1522-1-30.pdf?110908224221> (14.06.2013).

MÜLLER, Christian (2009): “Hochschule. Enorm expansionsfähig”. In: GaTE Germany (Hg.): Länderprofile Analysen- Erfahrungen- Trends. Edition Brasilien, 11-14. <http://www. gate-germany.de/fileadmin/bilder/Expertenwissen/laenderprofile_brasilien.pdf $>$ (14.06.2013). 
OECD (2010): PIS A 2009 Results: What Students Know and Can Do - Student Performance in Reading, Mathematics and Science, 1. Verfügbar unter: <http://www.oecd.org/pisa/pisaproducts/48852548.pdf> (13.06.2013).

- (2012): Education at a Glance: OECD Indicators 2012. BRAZIL.

Perez, Aparecida/Fraga de Melo, Gilberto/Fichtner, Bernd (2010): "Brasilien - ein Land mit Zukunft (?). Bildungssystem und alternative Bildungspraktiken". In: Costa, Sérgio/Kohlhepp, Gerd/Nitschack, Horst/Sangmeister, Hartmut (Hg.): Brasilien heute. Geographischer Raum, Politik, Wirtschaft, Kultur. 2. vollständig neu bearbeite Auflage. Frankfurt am Main: Vervuert, 623-636.

Richter, Claudia (2013): "Das Bildungswesen in Brasilien”. In: Adick, Christel (Hg.): Bildungsentwicklungen und Schulsysteme in Afrika, Asien, Lateinamerika und der Karibik. Münster: Waxmann, 171-190.

Schwartzman, Simon (2013): "Affirmative Action in Higher Education in Brazil: São Paulo's turn”. In: Blog from Center for International Higher Education. Washington. Verfügbar unter: <http://www.insidehighered.com/blogs/world-view/affirmative-action-higher-education-brazil-s \%C3\%A3o-paulo $\%$ E2\%80\%99s-turn> (13.06.2013).

The ECONOMIST (2012): “Coming soon: the world's priciest classrooms”. Verfügbar unter: <http://www.economist.com/blogs/americasview/2012/10/education-spendingbrazil $>(14.06 .2013)$.

UNESCO (2010): Word Data on Education. VII Ed. 2010/11. Brazil. Verfügbar unter: <http://www.ibe.unesco.org/fileadmin/user_upload/Publications/WDE/2010/ pdf-versions/Brazil.pdf> (14.06.2013).

Veras SoAres, Fabio/Perez Ribas, Rafael/Guerreiro Osório, Rafael (2007): Evaluating the Impact of Brazil's Bolsa Familia: Cash Transfer Programms in Comparative Perspective. Brasília: International Poverty Centre. Verfügbar unter: < http://www.ipc-undp.org/pub/ IPCEvaluationNote1.pdf> (05.06.2013).

WABER, Jörg (2007): "Hochschulzugang und Disparitäten. Deutsches und brasilianisches Bildungssystem im Vergleich". In: Tópicos, 3, 44-45. Verfügbar unter: <http://www. topicos.de/fileadmin/pdf/2007/3/k_waber.pdf> (14.06.2013). 